\title{
Direct Measurement of the Chudakov Effect
}

\author{
T. Virkus, ${ }^{1}$ H. D. Thomsen, ${ }^{1}$ E. Uggerh $\varnothing j,{ }^{1}$ U. I. Uggerh $ø j,{ }^{1}$ S. Ballestrero, ${ }^{2}$ P. Sona, ${ }^{2}$ A. Mangiarotti, ${ }^{3}$ T. J. Ketel, ${ }^{4}$
} A. Dizdar, ${ }^{5}$ S. Kartal, ${ }^{5}$ and C. Pagliarone ${ }^{6}$

(CERN NA63 Collaboration)

\author{
${ }^{1}$ Department of Physics and Astronomy, University of Aarhus, Denmark \\ ${ }^{2}$ University of Florence, Florence, Italy \\ ${ }^{3}$ Universidade de Coimbra, Coimbra, Portugal \\ ${ }^{4}$ Free University, Amsterdam, The Netherlands \\ ${ }^{5}$ University of Istanbul, Istanbul, Turkey \\ ${ }^{6}$ University of Cassino and INFN Pisa, Italy
}

(Received 29 November 2007; published 24 April 2008)

\begin{abstract}
Experimental results for the restricted energy loss of pairs created from 1-178 GeV photons in a thin $\mathrm{Au}$ target and subsequently passing a CCD detector are presented. It is shown that pairs - when detected close to the creation vertex - suffer a reduced energy loss due to the internal screening of the charges constituting the pair. Furthermore, the ability to measure directly the energy of the pair by calorimetry enables a comparison with theory as a function of energy. The observed phenomenon is in good qualitative agreement with general expectations from the Chudakov effect but indicates a quantitative disagreement with either of two mutually disagreeing theories.
\end{abstract}

DOI: 10.1103/PhysRevLett.100.164802

PACS numbers: 41.60.- m, 07.85.Fv, 29.40.Vj, 95.30.Gv

In the preparatory phase of the CERN NA63 experiment, we have investigated the reduced energy deposition from a positron-electron pair in the vicinity of their creation point. This reduction is due to the internal screening of charges, the so-called Chudakov (or King-Perkins-Chudakov) effect $[1,2]$.

By taking into account the density effect, a considerable contribution to the ionization energy loss originates from transverse distances $b_{q} \simeq v / \omega_{p}$, where $v$ is the particle speed through the medium with plasma frequency $\omega_{p}$. If a penetrating assembly of separate charges is internally spaced less than this distance, the ionization is influenced by interference terms. This can be the case, e.g., for an energetic hydrogen molecule that is stripped upon entry to the substance, but it can also be an effect present for an electron-positron pair where each participating charge screens the charge of the other as seen from the relevant distance $b_{q}$ in the medium. Because of the Lorentz transformation of angles to the laboratory system, the electron and positron emitted in the pair creation process from a photon of energy $\hbar \omega$ appear with an approximate angle of $1 / \gamma=m c^{2} / E_{e^{ \pm}} \simeq 2 m c^{2} / \hbar \omega$ to the photon momentum $\hbar \vec{k}$ in the frame of the laboratory. Thus, by defining $\xi_{ \pm}=$ $E_{e^{ \pm}} / \hbar \omega$ and $\gamma_{p}=\hbar \omega / m c^{2}$, we get an opening angle of the pair

$$
\phi \simeq 1 / \gamma_{p}\left[\xi_{-}\left(1-\xi_{-}\right)\right],
$$

the so-called Borsellino angle [3].

The energy loss thus diminishes close to the creation point if the created pair is sufficiently energetic and there- fore forward directed. This is the so-called Chudakov effect. In a sense, the Chudakov effect is the pair production analogue of the more familiar density effect in radiation emission.

A closely related effect - to both the density effect ([4], Chap. 13.5) and the Chudakov effect - has recently been calculated for Cherenkov radiation emission from $e^{+} e^{-}$ pairs in the vicinity of the creation point $[5,6]$. This internal screening effect may affect decisively the behavior of the Cherenkov emission in neutrino-induced electromagnetic showers. A similar reduction may apply in the case of vacuum-assisted photoionization [7], where the created pair that knocks out the electron may suffer internal screening, leading to a lower photoionization cross section. Finally, the radiation emission from relativistic positronium may be influenced by similar screening effects, depending on emission frequency [8].

As for the Landau-Pomeranchuk-Migdal effect-a disturbance of the projectile within the formation length that leads to a reduced cross section; see $[9,10]$, and references therein - the Chudakov effect has color transparency as an analogue in QCD [11].

Under the assumption that the created pair moves in a straight line after creation, the only angle that contributes to the separation is the emission angle $\simeq 1 / \gamma_{p}$, resulting in an opening angle of the pair $\simeq 4 m c^{2} / \hbar \omega$. A pair from a photon of energy $\hbar \omega$ would be separated by $b_{q}=v / \omega_{p}=$ $\beta c / \omega_{p}$ after having traversed a distance given by

$$
l_{s} \simeq \frac{4 \beta \hbar \omega}{m c \omega_{p}}
$$


such that, at distances smaller than $l_{s}$ from the creation point, interference between the constituents of the pair may arise. We note that $b_{q}$ is larger than the reduced Compton wavelength $\lambda_{c}=\hbar / m c \simeq 386 \mathrm{fm}$ - the transverse separation beyond which the pair can be considered created [12]—by a factor $m c^{2} / \hbar \omega_{p} \simeq 10^{4}$. Thus, the characteristic length scale for the pair formation zone is negligible in comparison to the length relevant for the Chudakov effect; i.e., the pair is real, but its charges are screening each other.

This mutual screening results in a reduced restricted energy loss at distances smaller than about $l_{s}$ from the creation vertex, showing the originally expected logarithmic dependence on separation [2]. The result from the destructive interference term from the opposite charges is a modified (restricted) energy loss of the pair $[13,14]$

$$
\frac{d E_{ \pm}}{d t}=2 \frac{\alpha \hbar \omega_{p}^{2}}{\beta}\left[\ln \left(\frac{\sqrt{2 m c^{2} T_{\mathrm{cut} / \mathrm{max}}}}{\hbar \omega_{p}}\right)-K_{0}\left(\frac{s \omega_{p}}{\beta c}\right)\right],
$$

where the first term corresponds to the usual restricted energy loss of two separate charges $e^{ \pm}$and the second term is due to the interference. Here $K_{0}(x)$ is the modified Bessel function of the second kind with order zero, and $s$ is the transverse separation of the pair which exceeds the longitudinal separation by a factor $\gamma$. The value for $T_{\text {cut }}=$ $10^{5} \mathrm{eV}$ is the energy at which the range of an electron corresponds to the active thickness of the detector, $16 \mu \mathrm{m}$ of $\mathrm{Si}$. As usual, the plasma frequency is given from $\omega_{p}^{2}=$ $4 \pi N Z e^{2} / m$, where $N Z$ is the number density of electrons.

For small separations $s \ll \beta c / \omega_{p} \simeq 64 \AA$ (in silicon), the modified Bessel function can be approximated by $K_{0}(x) \simeq \ln (1 / x)$, which results in

$$
\frac{d E_{ \pm}}{d t} \simeq 2 \frac{\alpha \hbar \omega_{p}^{2}}{\beta}\left[\ln \left(\frac{s \sqrt{2 m c^{2} T_{\mathrm{cut}}}}{\beta \hbar c}\right)\right]
$$

at small distances from the vertex. For large separations, the modified Bessel function tends exponentially to zero, corresponding to a loss of effective internal screening, and twice the standard expression for the stopping power for a single charge at high speed in a Fermi gas [see, e.g., [15], Eq. (5.165)] is retrieved from Eq. (3). We note that Eq. (4) breaks down at separations of the order of a Compton wavelength, but at this separation the pair can anyway not be considered "formed" [12], and classical electrodynamics is insufficient. It is perhaps a further indication of the limitations of the model that the expected energy loss dependence $d E / d x \propto s^{2}$ at small separations for an electric dipole [16] does not emerge from Eq. (4).

Clearly, according to Eq. (2), small plasma frequencies are preferable for the measuring device, so $\mathrm{Si}$ is a good material for the detection. On the other hand, since pair creation is roughly proportional to $Z^{2}$, it is advantageous to use high- $Z$ materials for the pair creation, of which gold is a good choice because of its structural properties.

There have been a few measurements of the Chudakov effect from cosmic ray tracks in emulsions $[2,17]$ - about a dozen in total. However, as previously noted [18], these measurements suffer from limited statistics and do not offer the possibility of investigating the effect in detail, since, e.g., the photon beam energy cannot be controlled nor measured directly.

The restricted, most probable energy loss (MPEL) is $\simeq 210 \mathrm{eV} / \mu \mathrm{m}$ in a thin silicon detector of thickness $\simeq 10 \mu \mathrm{m}$ [19]. With available beams of $\lesssim 100 \mathrm{GeV}$ photons, the relevant distances are $\lesssim 100 \mu \mathrm{m}$ to achieve a significant suppression according to Eq. (3). A thickness $\lesssim 20 \mu \mathrm{m}$ of both the conversion target and the detector itself is thus required to establish the condition that conversion takes place at a known distance (on the scale of $\lesssim 100 \mu \mathrm{m}$ ) and is unlikely to happen within the detector itself. If the latter happens, the pair that is created at, e.g., halfway through the detector will deposit only half the expected energy - even in the absence of the Chudakov effect - and therefore will mimic the searched effect. On the other hand, a detector thickness $\gtrsim 10 \mu \mathrm{m}$ is required to obtain a measurable signal (restricted energy loss larger than noise). For a detector of thickness $10 \mu \mathrm{m}$ and a maximum detector area $3 \mathrm{~mm}^{2}$, the noise is $\simeq 2 \mathrm{keV}$, to be compared to the signal per penetrating lepton, which is $2.1 \mathrm{keV}$. Typical GeV beams at CERN are a factor $\approx 1000$ larger in area, which means that using a "standard" solid state detector results in a very inefficient use of the beam, taking into account the noise.

In our experiment, we used instead a CCD detector (E2V CCD77-00-358) with an active layer thickness of $16 \mu \mathrm{m}, 512 \times 512$ pixels, a removable window, and a sensitive area of $12.3 \mathrm{~mm} \times 12.3 \mathrm{~mm}$, thus making efficient use of the beam. By grouping the columns, i.e., reading the CCD in 512 rows only, readout time could be reduced to less than $10 \mathrm{~ms}$, allowing for detection on an event-by-event basis. With the CERN beam of $\simeq 10^{4}$ $178 \mathrm{GeV}$ electrons per second during the $2.4 \mathrm{~s}$ long burst approximately 4 times a minute, this was done by use of a thin radiation target, $4.4 \% X_{0}$, ensuring "single photon conditions." A schematic setup is shown in Fig. 1. In short, the electron beam first passes a scintillator with a $\varnothing 9 \mathrm{~mm}$ hole $(\mathrm{ScH})$ that defines the beam; then the $0.125 \mathrm{~mm}$ thick Ir foil used to produce photons $(\mathrm{R})$ and is finally deflected in the magnetic dipole (B16) through a helium vessel (He). The produced photons pass (B16) and (He), after which a

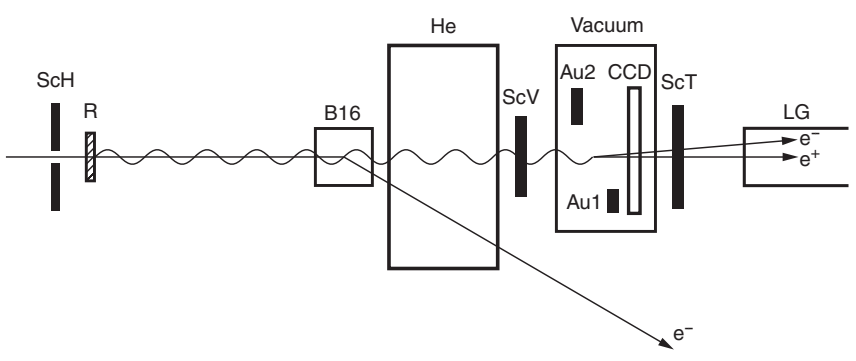

FIG. 1. Schematic setup of the Chudakov experiment at CERN. For details, see the text. 


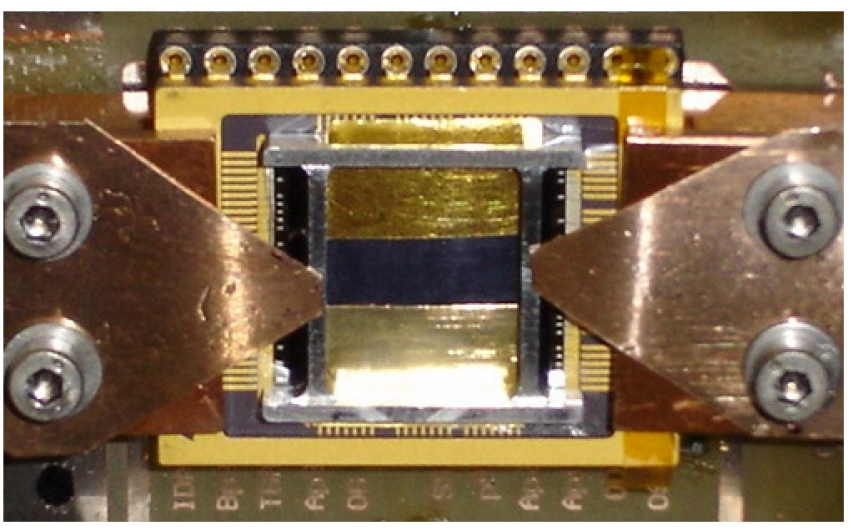

FIG. 2 (color online). A photograph of the CCD setup inside the vacuum chamber. To reduce dark current, the CCD was cooled to $\simeq 200 \mathrm{~K}$ by thermal contact to a liquid nitrogen reservoir through the $\mathrm{Cu}$ blocks shown.

veto scintillator $(\mathrm{ScV})$ with a minimal dead layer discards those events where the photon has converted upstream the vacuum chamber in which the CCD is located; see also Fig. 2. The entry and exit windows of the vacuum chamber are $22 \mu \mathrm{m}$ thick aluminized Mylar, and, on the downstream side, the trigger scintillator ( $\mathrm{ScT}$ ) contributes to the start of data taking if $\gtrsim 1.5$ minimum ionizing particles (MIPs) are detected. The $20 \mu \mathrm{m}$ thick Au target, $0.6 \% X_{0}$, gives a pair production probability 35 times higher than in the CCD. Finally, the photon and/or pair energy is determined from the downstream lead glass calorimeter (LG). The choice of a $20 \mu \mathrm{m}$ thick gold conversion target is a compromise between knowing the exact distance at which the pair is produced and the multiple scattering it introduces (favoring thin foils and low $Z$ ) and high conversion probability (favoring thick foils and high $Z$ ).

In the upper part of the CCD (see Fig. 2), the sequence of foils in the direction of the beam is (A): $20 \mu \mathrm{m} \mathrm{Au}$, $100 \mu \mathrm{m}$ Mylar, and $6 \mu \mathrm{m}$ Mylar; in the central part (B): $100 \mu \mathrm{m}$ Mylar and $6 \mu \mathrm{m}$ Mylar; and in the lower part (C): $100 \mu \mathrm{m}$ Mylar, $20 \mu \mathrm{m} \mathrm{Au}$, and $6 \mu \mathrm{m}$ Mylar. Since the CCD is read out as a function of the 512 available vertical positions (grouping the 512 horizontal channels in each case), the effective distance to the conversion vertex is given as $116 \pm 10 \mu \mathrm{m}$ for the upper channels and $16 \pm$ $10 \mu \mathrm{m}$ for the lower channels. In each case, and for each photon energy bin, a fit by a Landau distribution is performed to find the MPEL, i.e., the location of the peak of the distribution; see Fig. 3.

The uniformity of the response of the CCD detector and readout was investigated by use of an undeflected beam of electrons traversing the detector, thus giving rise to a restricted energy loss distribution with a MPEL corresponding to only a single charged particle. A comparison of the response in the CCD areas (A), (B), and (C) described above shows an excellent uniformity with $\operatorname{MPEL}_{(\mathrm{A})}=1.01 \pm 0.03 \mathrm{MPEL}_{(\mathrm{B})}=0.99 \pm 0.03 \mathrm{MPEL}_{(\mathrm{C})}$. Moreover, it is possible to compare the MPEL in

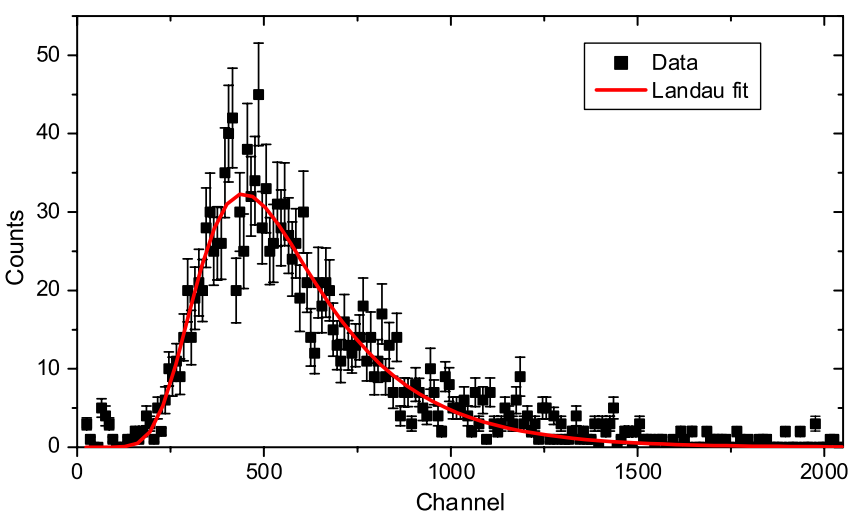

FIG. 3 (color online). A restricted energy loss spectrum as recorded by the CCD (squares with error bars) and a fit by a Landau distribution (line). The shown data set is for electrons penetrating the CCD.

section (A) for the pairs generated at an average distance of $116 \mu \mathrm{m}$ to the $\mathrm{CCD}_{\mathrm{MPEL}_{(\mathrm{A}, 2 e)}}$ to the energy loss of the single charged particle in the same section and for the same energy $\operatorname{MPEL}_{(\mathrm{A}, e)}$ obtained with B16 off. This ratio is somewhat lower than $2, \operatorname{MPEL}_{(\mathrm{A}, 2 e)}=1.62 \pm$ $0.08 \mathrm{MPEL}_{(\mathrm{A}, e)}$, and we have corrected for this systematic error by multiplying the data points by $2 / 1.62$, forcing the ratio $\operatorname{MPEL}_{(\mathrm{A}, 2 e)} / \operatorname{MPEL}_{(\mathrm{A}, e)}$ to be consistent with 2 . The expected suppression at a distance of $116 \mu \mathrm{m}$ according to $[13,14]$ is 0.998 . It is possible that the single charged particle is followed by a $\delta$ electron, but such events are rejected by the use of $\mathrm{ScV}$ and $\mathrm{ScT}$, requiring one charged particle only, and are not seen in Fig. 3. Alternatively, one of the created leptons of the pair may escape detection, being too distant from the other on the scale of the pixel size $24 \mu \mathrm{m}$. This may happen since only firings in adjacent rows are summed but is very unlikely since the average transverse distance between the $e^{+}$and $e^{-}$produced at the $116 \pm 10 \mu \mathrm{m}$ distant foil is less than $0.01 \mu \mathrm{m}$, including multiple scattering. We emphasize that, even though the exact origin and magnitude of the factor $2 / 1.62$ are uncertain, the quoted uncertainty being statistical only, the general trend of our experimental values is unaffected by this, and only the absolute scale is questionable to an accuracy of about $20 \%$.

In Fig. 4 are shown the experimental results (based on $2 \times 10^{7}$ electrons in total) for the ratio of the MPEL for $\mathrm{Au} 1$ to that of Au2, compared to three theoretical expectations. We note that, since this is a relative measurement, uncertainties connected to departures from an exact Landau shape [19] or a possible reduced energy deposition at high energies $[20,21]$ are likely to be insignificant. Such departures could - besides the thickness dependence - for instance, result from a smaller suppression at small impact parameters than at large ones, i.e., a smaller suppression at energy losses large compared to the MPEL. The dashed line shows the expected values based on Eq. (3), where the pair separation $s$ has been found from the opening angle, 


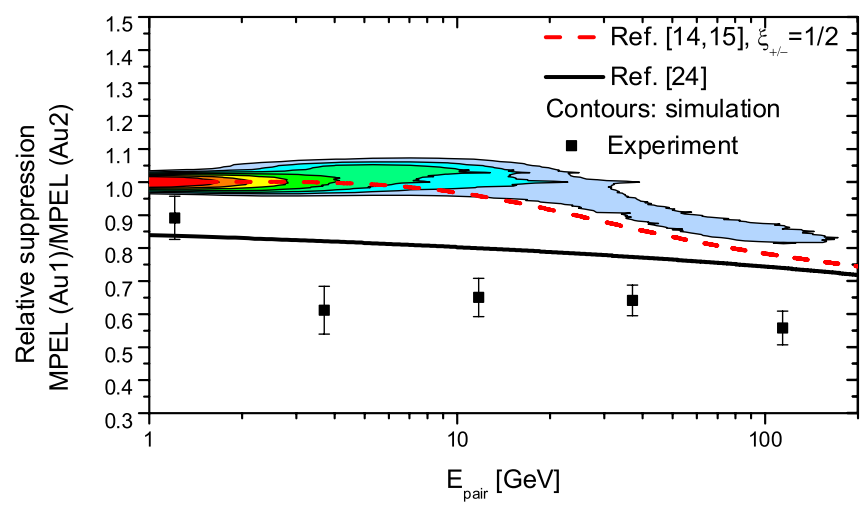

FIG. 4 (color online). The MPEL for the gold foil positioned at an average distance of $16 \mu \mathrm{m}$ from the CCD divided by the MPEL for the gold foil positioned at an average distance of $116 \mu \mathrm{m}$ from the CCD. The solid squares show the values obtained in the experiment, the dashed line shows the expected ionization reduction based on Eq. (3) with $\xi_{ \pm}=1 / 2$, and the contour plot represents simulated values as described in the text. The solid line represents the expected ionization reduction from a quantum treatment [24].

Eq. (1) with $\xi_{ \pm}=1 / 2$, and the distance traversed. The fact that the low energy point is close to 1 , as expected, gives confidence in the 2 MIP-1 MIP ratio correction.

As contours our simulation is shown, which includes (1) the angular distribution generated according to the Borsellino distribution [3], (2) the energy distribution $d N_{p} / d \xi_{ \pm} \propto\left(\xi_{+}^{2}+\xi_{-}^{2}+\frac{2}{3} \xi_{+} \xi_{-}\right) \quad$ [22], (3) multiple Coulomb scattering according to the standard expression [23], and (4) finite target thicknesses. An effect similar to the Chudakov effect is to be expected for multiple Coulomb scattering as well, but, since the relevant impact parameters are about 2 orders of magnitude smaller than $v / \omega_{p}$, such an effect is completely insignificant at the energies investigated here. The simulation is performed on the basis of the Chudakov effect expected from Eq. (3) $[13,14]$. A quantum treatment of the Chudakov effect is shown as a solid line [24]. However, the latter is different from one even at energies as low as $10 \mathrm{MeV}$, where it predicts a suppression to $88 \%$ for the setup considered.

As seen in Fig. 4, our experimental data points show a stronger effect than expected from theory. The measurement favors the quantum treatment of the Chudakov effect [24] compared to the (semi)classical $[13,14]$. However, the theory of [24] has a severe drawback in producing a suppression factor larger than 1 for pairs with a total energy smaller than about $180 \mathrm{GeV}$, generated in the $116 \pm$ $10 \mu \mathrm{m}$ distant foil. Only the ratio of the MPELs for the two foils becomes well defined. A more firm conclusion concerning which theoretical model would be favored would require better statistics and a more careful calibration of the 2 MIP-1 MIP ratio, but as noted above the theoretical approaches also suffer from inadequacies. The data points are generally reduced by about $5 \%$ for a high trigger threshold $(\gtrsim 2)$ MIP, but they also become much more uncertain due to reduced statistics. Nevertheless, it has been shown that it is possible to measure the Chudakov effect directly (i.e., from the actual ionization energy loss, not through blob densities in nuclear emulsions) in an accelerator environment with the advantages that this gives on, e.g., a similarly direct measurement (by calorimetry instead of pair opening angles) of pair energies. Furthermore, the Chudakov effect has been shown to contribute substantially to the decrease of restricted energy loss for detectors close to the creation vertex, an effect that is significant-even up to distances of a millimeter - at energies relevant for the LHC.

We acknowledge the strong support from P.B. Christensen and P. Aggerholm (DPA, Aarhus) as well as I. Efthymiopoulos and A. Fabich (CERN). Thanks are due to E. Lægsgaard and S.P. Møller for useful comments on energy losses in thin Si detectors.

[1] A. E. Chudakov, Izv. Akad. Nauk SSSR, Ser. Fiz. 19, 650 (1955) [Bull. Acad. Sci. USSR, Phys. Ser. 19, 589 (1955)].

[2] D. H. Perkins, Philos. Mag. 46, 1146 (1955).

[3] A. Borsellino, Phys. Rev. 89, 1023 (1953).

[4] J.D. Jackson, Classical Electrodynamics (Wiley, New York, 1975).

[5] L. A. Gevorgian, K. A. Ispirian, M. K. Ispirian, and F. Piuz, Europhys. Lett. 34, 183 (1996).

[6] S. K. Mandal, S. R. Klein, and J. D. Jackson, Phys. Rev. D 72, 093003 (2005).

[7] A. Belkacem and A. H. Sørensen, Radiat. Phys. Chem. 75, 656 (2006).

[8] U. I. Uggerhøj, Phys. Rev. A 73, 052705 (2006).

[9] S. Klein, Rev. Mod. Phys. 71, 1501 (1999).

[10] H. D. Hansen, U. I. Uggerhøj, C. Biino, S. Ballestrero, A. Mangiarotti, P. Sona, T. J. Ketel, and Z. Z. Vilakazi, Phys. Rev. D 69, 032001 (2004).

[11] L. L. Frankfurt, G. A. Miller, and M. Strikman, Annu. Rev. Nucl. Part. Sci. 44, 501 (1994).

[12] U. I. Uggerhøj, Rev. Mod. Phys. 77, 1131 (2005).

[13] A.I. Akhiezer and N.F. Shul'ga, High Energy Electrodynamics in Matter (Gordon and Breach, New York, 1996).

[14] V. B. Berestetskii and B. V. Geshkenbain, Sov. Phys. JETP 4, 609 (1957).

[15] P. Sigmund, Particle Penetration and Radiation Effects (Springer, New York, 2006).

[16] N. R. Arista, Phys. Rev. A 65, 022902 (2002).

[17] P. L. Jain, Phys. Rev. 125, 679 (1962).

[18] I. P. Zielinski, Nucl. Instrum. Methods Phys. Res., Sect. A 238, 562 (1985).

[19] J. F. Bak et al., Nucl. Phys. B288, 681 (1987).

[20] H. Bichsel, Rev. Mod. Phys. 60, 663 (1988).

[21] W. Ogle, P. Goldstone, C. Gruhn, and C. Maggiore, Phys. Rev. Lett. 40, 1242 (1978).

[22] W. Heitler, The Quantum Theory of Radiation (Dover, New York, 1954).

[23] W.-M. Yao et al., J. Phys. G 33, 1 (2006).

[24] G. H. Burkhardt, Nuovo Cimento 9, 375 (1958). 